\title{
Cervical Spine Disability in Correlation with Subjective Voice Handicap in Patients With Voice Disorders: A Retrospective Analysis
}

\author{
Langenfeld, Anke ; Bohlender, Jörg E ; Swanenburg, Jaap ; Brockmann-Bauser, Meike
}

\begin{abstract}
OBJECTIVE Neck muscle dysfunction has been considered as risk factor or consequence of voice disorders. This study investigates the correlation between neck and voice-related subjective symptoms in patients with voice disorders. STUDY DESIGN Retrospective case-control study. METHODS 100 adult patients (59 female and 41 male) over 18 years with a mean age of 50.01 years (SD 16, range 24-87), of which 68 were under 60 years, were included. 50 patients had organic voice pathologies and 50 functional dysphonia. Outcome measures were the Voice Handicap Index 9 international (VHI-9i) and the Neck Disability Index (NDI-G). Spearman rank order coefficient was applied to determine the correlation between overall and single item VHI and NDI results. Subanalyses were done for functional vs. organic disorder, gender and age \pm 60 years. RESULTS Mean overall VHI-9i (13.93, SD = 7.81, range $=0-31)$ and mean NDI-G $(6.07, \mathrm{SD}=7.71$, range $=0-43)$ showed a significant mild correlation $(\mathrm{r}$ $=0.220, \mathrm{P}=0.02)$. Split into subgroups the relation was stronger in patients with organic pathologies $(\mathrm{r}$ $=0.297, \mathrm{P}=0.03)$, but not significant in functional disorders $(\mathrm{r}=0.148, \mathrm{P}=0.30)$. There was a moderate relation in men $(\mathrm{r}=0.317, \mathrm{P}=0.04)$. Single item correlation was highest between VHI-9i item P4 (physiological) and NDI-G item reading (cognitive functioning) $(\mathrm{r}=0.480, \mathrm{P}=0.002)$. CONCLUSION Specifically patients with organic voice disorders showed increased voice symptoms with the presence of neck dysfunction. This indicates a risk for a functional imbalance of the muscles surrounding the larynx, which in extreme cases may hinder functional voice rehabilitation even after phonosurgery. Therefore, neck dysfunction should be considered in voice diagnostics.
\end{abstract}

DOI: https://doi.org/10.1016/j.jvoice.2018.10.013

Posted at the Zurich Open Repository and Archive, University of Zurich ZORA URL: https://doi.org/10.5167/uzh-166608

Journal Article

Accepted Version

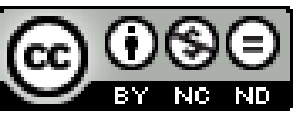

The following work is licensed under a Creative Commons: Attribution-NonCommercial-NoDerivatives 4.0 International (CC BY-NC-ND 4.0) License.

Originally published at:

Langenfeld, Anke; Bohlender, Jörg E; Swanenburg, Jaap; Brockmann-Bauser, Meike (2020). Cervical Spine Disability in Correlation with Subjective Voice Handicap in Patients With Voice Disorders: A Retrospective Analysis. Journal of Voice, 34(3):371-379.

DOI: https://doi.org/10.1016/j.jvoice.2018.10.013 


\section{Cervical Spine Disability in Correlation with Subjective Voice Handicap in Patients With Voice Disorders: A Retrospective Analysis}

\section{Anke Langenfeld $*$ + \\ Jörg E. Bohlender*}

Jaap Swanenburg $\dagger+$

Meike Brockmann-Bauser*,

Department of Phoniatrics and Speech Pathology, Clinic for Otorhinolaryngology, Head and Neck Surgery, University Hospital Zurich, University of Zurich, Zurich, Switzerland

†Physiotherapy Occupational Therapy Research, Directorate of Research and Education, University Hospital Zurich, Zurich, Switzerland

\#Integrative Spinal Research ISR, Department of Chiropractic Medicine, Balgrist University Hospital, Zurich, Switzerland

Address correspondence and reprint requests to Meike Brockmann-Bauser, Department of Phoniatrics and Speech Pathology, Clinic for Otorhinolaryngology, Head and Neck Surgery, University Hospital Zurich, University of Zurich, Frauenklinikstrasse 24, 8091 Zurich, Switzerland.

\section{Abstract \\ Objective}

Neck muscle dysfunction has been considered as risk factor or consequence of voice disorders. This study investigates the correlation between neck and voice-related subjective symptoms in patients with voice disorders.

Study Design

Retrospective case-control study.
Please confirm that givennames and surnames have been identified correctly.

\section{Reply $\bigcirc$ Yes $\bigcirc$ No}

Yes

Attach (optional)

Limit $20 \mathrm{MB}$ /file

Corrections were submitted via Proof Central (Online method) for this article. No further modification can be made via this interface. 


\section{Methods}

100 adult patients ( 59 female and 41 male) over 18 years with a mean age of 50.01 years (SD 16, range 24-87), of which 68 were under 60 years, were included. 50 patients had organic voice pathologies and 50 functional dysphonia. Outcome measures were the voice handicap index 9 international (VHI-9i) and the neck disability index (NDI-G). Spearman rank order coefficient was applied to determine the correlation between overall and single item VHI and NDI results. Subanalyses were done for functional vs organic disorder, gender and age \pm 60 years.

\section{Results}

Mean overall VHI-9i (13.93, SD = 7.81, range $=0-31)$ and mean NDI-G (6.07, SD = 7.71, range $=0-43)$ showed a significant mild correlation $\left(\mathrm{r}_{\mathrm{s}}=0.220, P=0.02\right)$. Split into subgroups the relation was stronger in patients with organic pathologies $\left(\mathrm{r}_{\mathrm{s}}=0.297, P=0.03\right)$, but not significant in functional disorders $(\mathrm{r}=0.148, P=0.30)$. There was a moderate relation in men $\left(\mathrm{r}_{\mathrm{s}}=0.317\right.$, $P=0.04$ ). Single item correlation was highest between VHI-9i item P4 (physiological) and NDI-G item reading (cognitive functioning) $\left(\mathrm{r}_{\mathrm{s}}=0.480, P=0.002\right)$.

\section{Conclusion}

Specifically patients with organic voice disorders showed increased voice symptoms with the presence of neck dysfunction. This indicates a risk for a functional imbalance of the muscles surrounding the larynx, which in extreme cases may hinder functional voice rehabilitation even after phonosurgery. Therefore, neck dysfunction should be considered in voice diagnostics.

Key Words: Voice disorders; Voice diagnostics; Voice handicap index (VHI); Neck pain; Neck disability index (NDI)

\section{Introduction}

Around $30 \%$ of the general population suffers from a clinically relevant voice disorder once during the individual's lifetime. The lifetime prevalence in professional voice users such as teachers may be around $57 \%$, and thus substantially higher as compared low voice users. ${ }^{1-5}$ Voice disorders may be caused from changes in the innervation, structure or function of the phonatory system. ${ }^{6}$ Organic voice disorders refer to restrictions of voice quality or vocal performance due to structural alterations of the vocal folds or neurogenic causes, whereas so-called functional voice disorders are characterized by the absence of structural and neurogenic etiologies. ${ }^{7}$ Functional voice disorders have been discussed as underlying cause of structural 
findings such as vocal fold nodules or cysts, and also as secondary adaptation phenomenon to laryngeal pathology. 8

Functional voice disorders, also termed as muscle tension dysphonia or behavioral dysphonia, are diagnosed in around $30 \%$ of patients in voice clinics. ${ }^{6}$ Most patients describe voice-related physical, functional and emotional subjective symptoms, which can be evaluated by standardized questionnaires such as the voice handicap index (VHI). ${ }^{3,9}$ Furthermore, especially in professional voice users, a functional voice disorder may lead to extended sick leaves and considerable psychological distress. ${ }^{5}$ As underlying causes for functional voice disorders, an inadequate voice technique resulting from a lack of training, high vocal demand, working conditions, personality characteristics, and psychological disorders have been discussed. ${ }^{8,10}$ In voice clinics these patients may present with hypertension of the laryngeal, paralaryngeal, ${ }^{8}$ pharyngeal, tongue, neck and respiratory musculature. ${ }^{8}$ As a consequence, inadequate vocal behavior associated with hyperfunction of the neck muscles may lead to pain within the shoulder girdle and cervical region. ${ }^{10-12}$ As Silverio et al (2014) reported in a study of 30 women with dysphonia, these had a statistically significant higher frequency of neck pain as compared to 30 nondysphonic women. ${ }^{13}$ Furthermore, pain intensity at the back of the neck was significantly higher than in other areas of the body. ${ }^{10,13}$

In turn it has been suggested that neck pain is one risk factor, which may contribute to the development of voice disorders. ${ }^{14}$ Neck pain, in conjunction with low back pain, is one of the leading causes for disability in high-income countries. ${ }^{15}$ People who experienced neck pain once are likely to have more occurrences during their lifetime, because relapses are common and often no definitive curative treatment can be found. ${ }^{16}$ Further, the location of the pain does not imply that the underlying cause of the symptom has its origin within this area. ${ }^{17}$ Neck pain and disability is often accompanied by concomitant pains such as headache, shoulder, upper limb, upper back and/or low back pain. ${ }^{18,19}$ Considering the role of neck and throat area muscles in head and shoulder movements, which also influence the position of the larynx, an effect of neck pain or dysfunction toward functional or even benign structural voice disorders is possible. ${ }^{10}$ As shown in studies examining the effects of flow-resistant tubes in semioccluded vocal tract voice training programs on voice function, the position of the larynx may play a key role in vocal efficiency. ${ }^{20}$ Further, Mathieson proposed based on a literature review, that manual therapy may be an efficient primary treatment approach in patients with functional dysphonia. ${ }^{21}$ In voice clinics it often remains unclear which complaint, functional voice, or neck-related symptoms, develops first and if or how they interact. Therefore, the objective of the present study was to evaluate if patients with functional and organic voice disorders suffer from neck-related handicaps during daily life activities, and if these correlate with subjective voice handicap. 


\section{Methods}

\section{Study design and participants}

The present study was done in a retrospective case-control design. Patients having an appointment at the Department of Phoniatrics and Speech Pathology, Clinic for Otorhinolaryngology, Head and Neck Surgery, University Hospital Zurich, Switzerland, were asked beforehand if they agree that data collected during their visit may be used for retrospective analysis. Suitable participant data was selected from the hospital`s patient management system. All diagnoses were determined applying the diagnostic protocol of the European Laryngological Union, and included a visual, subjective, perceptual, instrumental acoustic and aerodynamic assessment. ${ }^{22}$ Ethical approval was granted from the responsible ethics board under reference number 2016-00455 and is registered at Clinicaltrials.gov under Nr. NCT02868762.

\section{Exclusion criteria}

Patients had to be over 18 years old and to be diagnosed with either a functional or organic voice disorder such as vocal fold cyst, pseudo cysts, granuloma, polyps, Reinke`s edema, or posterior laryngitis (Table 1). Patients were excluded if they had any systemic diseases such as rheumatism, a recent history of cancer, neurogenic disease (such as Parkinson`s disease), or spine surgery causing vocal fold motion impairment or immobility, or have had an intubation within the previous 18 months. Patients unable to fill out questionnaires due to language or cognitive issues were also not included.

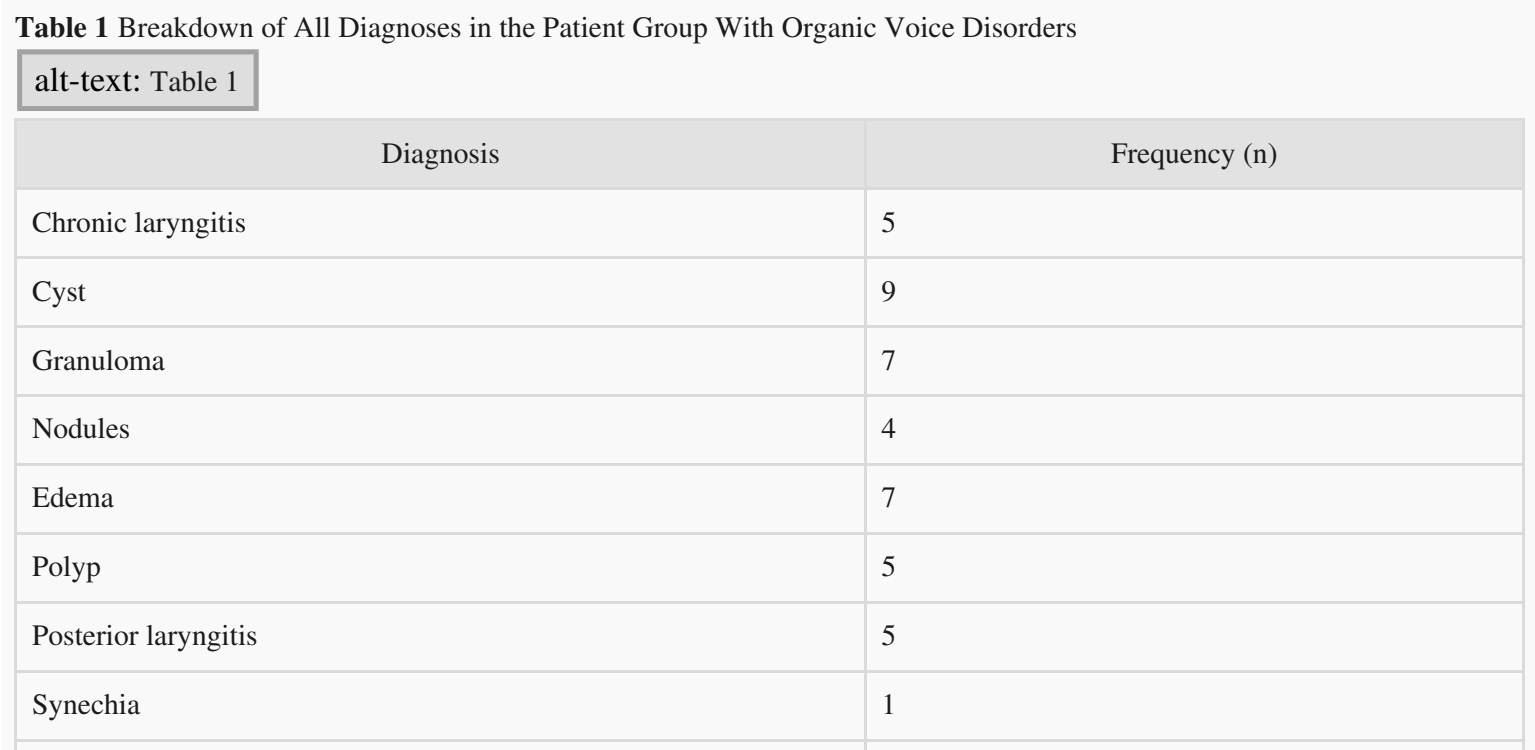




\begin{tabular}{|l|l|}
\hline Scars & 3 \\
\hline Peripheral VFMI & 3 \\
\hline Sulcus vocalis & 1 \\
\hline Total & 50 \\
\hline
\end{tabular}

Peripheral VFMI, vocal fold motion impairment due to peripheral lesions of the recurrent laryngeal nerve.

\section{Outcome measures}

Main outcome measures were the German version of the voice handicap index 9 international (VHI9i, Appendix 1) and the German version of the neek disability index-Please write: Neck Disability Index(NDIG, Appendix 2), both are described in detail below. Additional data regarding age, gender, and diagnosis was collected for each patient. All measurements were taken before or during the first appointment before treatment at the Department for Phoniatrics and Speech Pathology at the Clinic for Otorhinolaryngology, Head and Neck Surgery, University Hospital Zurich, Zurich, Switzerland.

\section{Voice handicap index 9 international}

The German version of the VHI-9i is a self-report questionnaire with established clinical validity and reliability ${ }^{23-26}$ to measure the severity of specific subjective, functional, and emotional voice-related symptoms. ${ }^{24}$ It consists of nine questions (items): items F1, F3, F5, and F16 cover functional, item P4, P17 and P21 physiological and item E24 and E29 emotional components (Appendix 1). Each item scores up to four on a four point Likert scale (0-4), with a maximum overall score of 36. Higher scores are associated with more subjective voice-related symptoms. ${ }^{27}$ A score of $0-5$ indicates no, a score of 6-10 a mild, a score of 11-16 a moderate and a score above 17 a severe voice-related handicap. ${ }^{24}$

\section{Neek disability index (NDI-G)}

The neck disability index has been described as one of the most widely used questionnaires to assess neck-related subjective disability with excellent psychometric properties. ${ }^{28}$ It is a short paper-pencil questionnaire, which is based on a similar instrument used for patients with low back pain, the Oswestry Low Back Pain Questionnaire. ${ }^{29}$ It consists of 10 items related to activities of daily living and has a two factor structure $^{30}$ : One factor assesses pain and functional disability (items 1, 2, 3, 4, 7, 10), and the other factor cognitive functioning (items 5, 6, 8, 9, Appendix 2). Each item scores up to five on a Likert scale with a total score of 50. The lower the score, the less is the subjectively perceived disability. ${ }^{31}$ In the present study we applied the NDI-G by Swanenburg et al. (2014). ${ }^{30}$ 


\section{Data analysis and statistics}

Descriptive statistics including age to describe the patient characteristics using SPSS Version 21 statistical software package (SPSS, IBM Inc., Chicago, IL). Nonparametric statistics were used for inferential data analysis, because both outcome measures were given in ordinal scales. Spearman-Rank-Order Correlation $\left(r_{s}\right)$ was applied to measure correlations between both questionnaires. The correlation coefficient $r_{s}$ is a number between -1 and $+1,32$ while numbers between 0.10 and 0.29 refer to a weak, 0.30 and 0.49 to a moderate, and between 0.50 and 1.0 to a large correlation. ${ }^{33}$ Furthermore, the sample was split into the subgroups functional vs organic, male vs female, and patients over 60 years old vs patients under 60 years old to determine systematic differences.

\section{Results \\ Descriptive characteristics \\ Examined group}

100 patients between 24 and 87 years with a mean age of 50.01 years (SD 16) were included. Of these, 59 were female and 41 male, and 68 were younger than 60 years of age. Fifty patients were diagnosed with muscle tension dysphonia (27 female/ 23 male) and 50 with organic voice disorder (32 female/18 male). Please find a detailed breakdown of diagnoses in Table 1.

\section{VHI-9i and NDI-G}

Mean overall VHI-9i score was 13.93 (moderate, $\mathrm{SD}=7.81$, range $=0-31$ ) and mean NDI-G score was 6.07 (mild, SD 7.71, range 0-43). According to VHI-9i 36.6\% $(\mathrm{n}=15)$ of the male participants had mild to moderate disability, and $48.8 \%(\mathrm{n}=20)$ had severe disability. In the female participants $38.9 \%(\mathrm{n}=24)$ had mild to moderate disability, and $42.4 \%(n=25)$ had severe disability (Figure 1$)$. 


\section{VHI-9i and NDI-G disability ratings in the whole group after gender}
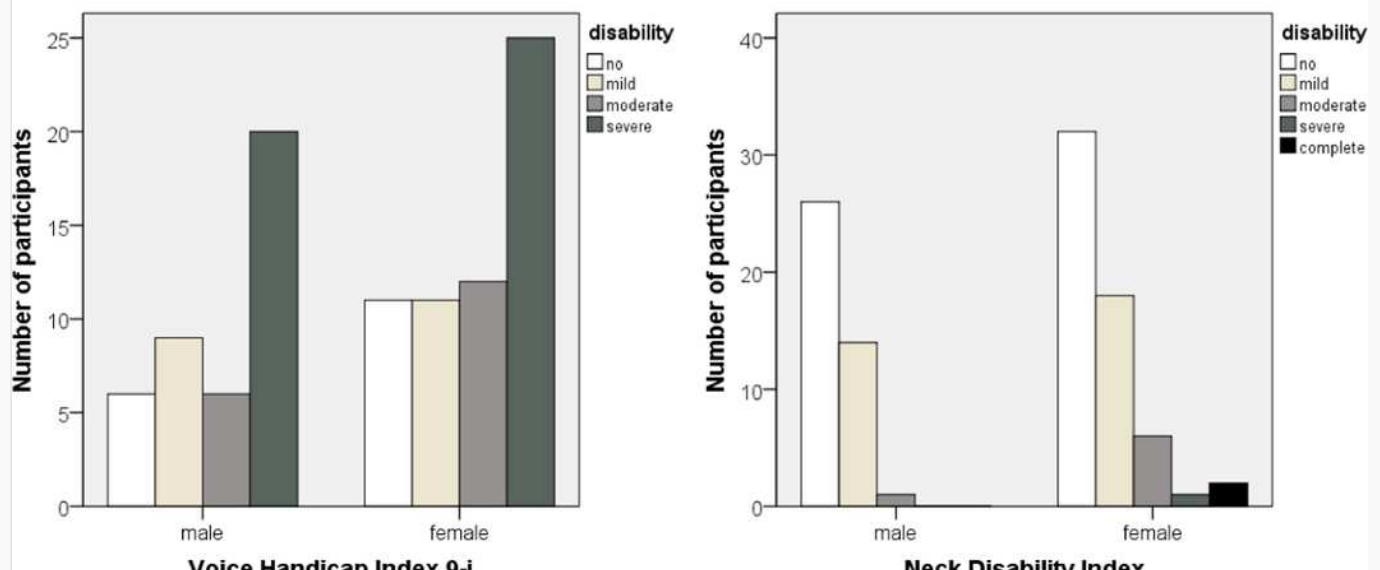

Figure 1 Bar charts indicating VHI-9i (left) and NDI-G (right) subjective handicap severity rating distributions in the whole examined group of 100 voice patients for women $(n=59)$ and men $(n=41)$. alt-text: Figure 1.

Referring to the NDI-G 63.4\% $(\mathrm{n}=26)$ male participants had no disability, $34.1 \%(\mathrm{n}=14)$ had mild, $2.4 \%(\mathrm{n}=1)$ moderate, and none had severe disability. $54.2 \%(\mathrm{n}=32)$ of female participants had no disability, $30.5 \%(n=18)$ had mild, $10.2 \%(n=6)$ moderate, $1.7 \%(n=1)$ severe and $3.4 \%(n=2)$ had complete disability (Figure 1).

\section{Total sample correlations between VHI-9i and NDI-G}

There was a significant weak correlation between both questionnaires` total scores $(r=.220$, $P=0.02)$. In total, there were 16 single item correlations. Strongest correlations were found between physiological item 17 and Personal care $(\mathrm{r}=0.309, P=0.002)$, physiological items 4 and 17 and Headaches (P4: $\mathrm{r}=0.325, P=0.001 ; \mathrm{P} 17: \mathrm{r}=0.281, P=0.005)$, physiological item 4 and Concentration $(\mathrm{r}=0.308$, $P=0.002)$, emotional item 29 and Personal Care $(\mathrm{r}=0.270, P=0.007)$ and physiological item 4 and 17 and Recreation $(\mathrm{P} 4 \mathrm{r}=0.288, P=0.004 / \mathrm{P} 17 \mathrm{r}=0.281, P=0.005)$. Table 2 summarizes all correlations for the total sample. 


\begin{tabular}{|c|c|c|c|}
\hline Item VHI & $\begin{array}{c}\text { Item NDI } \\
\text { (Factor) }\end{array}$ & $r_{s}$ & $P$ Value \\
\hline$F 3$ & $\begin{array}{l}\text { Reading } \\
\text { (PFD) }\end{array}$ & 0.201 & 0.048 \\
\hline P17 & $\begin{array}{l}\text { Personal care } \\
\text { (PFD) }\end{array}$ & 0.309 & $0.002 * *$ \\
\hline$P 17$ & $\begin{array}{l}\text { Reading } \\
\text { (PFD) }\end{array}$ & $\mathbf{0 . 2 5 7}$ & $\mathbf{0 . 0 1 0}$ \\
\hline$P 4$ & $\begin{array}{l}\text { Headaches } \\
\text { (CF) }\end{array}$ & 0.325 & $0.001 * *$ \\
\hline F16 & $\begin{array}{l}\text { Headaches } \\
(\mathrm{CF})\end{array}$ & 0.234 & 0.016 \\
\hline$P 17$ & $\begin{array}{l}\text { Headaches } \\
(\mathrm{CF})\end{array}$ & 0.281 & $0.005 * *$ \\
\hline P4 & $\begin{array}{l}\text { Concentration } \\
\text { (CF) }\end{array}$ & 0.308 & $0.002 * *$ \\
\hline$E 24$ & $\begin{array}{l}\text { Personal care } \\
\text { (PFD) }\end{array}$ & 0.230 & 0.021 \\
\hline E29 & $\begin{array}{l}\text { Personal care } \\
\text { (PFD) }\end{array}$ & 0.270 & $0.007 * *$ \\
\hline E24 & $\begin{array}{l}\text { Reading } \\
\text { (PFD) }\end{array}$ & 0.226 & 0.024 \\
\hline E24 & $\begin{array}{l}\text { Concentration } \\
\text { (CF) }\end{array}$ & 0.209 & 0.037 \\
\hline E29 & $\begin{array}{l}\text { Concentration } \\
(\mathrm{CF})\end{array}$ & 0.238 & 0.017 \\
\hline$P 4$ & $\begin{array}{l}\text { Driving } \\
\text { (CF) }\end{array}$ & 0.256 & 0.010 \\
\hline P4 & $\begin{array}{l}\text { Recreation } \\
\text { (PFD) }\end{array}$ & 0.288 & $0.004 * *$ \\
\hline F16 & $\begin{array}{l}\text { Recreation } \\
\text { (PFD) }\end{array}$ & 0.234 & 0.021 \\
\hline P17 & $\begin{array}{l}\text { Recreation } \\
\text { (PFD) }\end{array}$ & 0.281 & $0.005^{* *}$ \\
\hline
\end{tabular}

16 correlations were found ranging between 0.201 (small effect) and 0.325 (medium effect). The strongest statistical

correlation was found for questions $\mathrm{P} 4$ and headaches $(0.325)$. VHI-9i subscales: $\mathrm{E}=$ emotional, $\mathrm{P}=$ physiological,

$\mathrm{F}=$ functional. $\mathrm{NDI}-\mathrm{G}$ factors: $\mathrm{CF}=$ cognitive functioning, $\mathrm{PFD}=$ pain and functional disability. $* *$ Correlation is significant at the 0.01 level. * Correlation is significant at the 0.05 level. 


\section{Subgroup correlations}

\section{Diagnosis}

Patients with organic voice disorders had a statistically significant but weak (almost moderate) correlation between the NDI-G and VHI-9i $(r=0.297, P=0.03)$. For patients with functional voice disorders alone, there was a weak, not statically significant correlation $(\mathrm{r}=0.148, P=0.30)$. In the subgroup of patients with organic voice disorders there were 19 statistical significant single item correlations ranging from 0.290 (weak) to 0.457 (moderate). The strongest and moderate correlations were found between the items "work" and E29 (r = 0.401, $P=0.044)$, "personal care" and E29 (r = 0.407, $P=0.003)$, "recreation" and P17 $(\mathrm{r}=0.415$, $P=0.003)$, "headaches" and P17 $(\mathrm{r}=0.425, P=0.002)$ and "headaches" and F16 $(\mathrm{r}=0.457, P=0.001)$. For a complete overview of the organic subgroup please see Table 3.

\begin{tabular}{|c|c|c|c|}
\hline alt-text: Table 3 & & & \\
\hline Item VHI & Item NDI & $r_{s}$ & $P$ Value \\
\hline$F 3$ & $\begin{array}{l}\text { Reading } \\
\text { (PFD) }\end{array}$ & 0.370 & 0.010 \\
\hline F3 & $\begin{array}{l}\text { Headaches } \\
\text { (CF) }\end{array}$ & 0.374 & 0.010 \\
\hline$F 3$ & $\begin{array}{l}\text { Concentration } \\
\text { (CF) }\end{array}$ & 0.317 & 0.030 \\
\hline$F 3$ & $\begin{array}{l}\text { Sleeping } \\
\text { (CF) }\end{array}$ & 0.290 & 0.048 \\
\hline F16 & $\begin{array}{l}\text { Reading } \\
\text { (PFD) }\end{array}$ & 0.322 & 0.024 \\
\hline$P 4$ & $\begin{array}{l}\text { Headaches } \\
\text { (CF) }\end{array}$ & 0.339 & 0.016 \\
\hline F16 & $\begin{array}{l}\text { Headaches } \\
(\mathrm{CF})\end{array}$ & 0.457 & $0.001 * *$ \\
\hline F16 & $\begin{array}{l}\text { Concentration } \\
\text { (CF) }\end{array}$ & 0.369 & 0.009 \\
\hline F16 & $\begin{array}{l}\text { Recreation } \\
\text { (PFD) }\end{array}$ & 0.338 & 0.017 \\
\hline E24 & $\begin{array}{l}\text { Personal Care } \\
\text { (PFD) }\end{array}$ & 0.329 & 0.020 \\
\hline
\end{tabular}




\begin{tabular}{|c|c|c|c|}
\hline$P 17$ & $\begin{array}{l}\text { Headaches } \\
\text { (CF) }\end{array}$ & 0.425 & $0.002 * *$ \\
\hline$E 24$ & $\begin{array}{l}\text { Headaches } \\
\text { (CF) }\end{array}$ & 0.292 & 0.040 \\
\hline$E 29$ & $\begin{array}{l}\text { Work } \\
\text { (PFD) }\end{array}$ & 0.303 & 0.032 \\
\hline P17 & $\begin{array}{l}\text { Recreation } \\
\text { (PFD) }\end{array}$ & 0.415 & $0.003^{* *}$ \\
\hline$E 24$ & $\begin{array}{l}\text { Recreation } \\
\text { (PFD) }\end{array}$ & 0.290 & 0.041 \\
\hline$E 29$ & $\begin{array}{l}\text { Personal Care } \\
\text { (PFD) }\end{array}$ & 0.407 & $0.003 * *$ \\
\hline$E 29$ & $\begin{array}{l}\text { Lifting } \\
\text { (PFD) }\end{array}$ & 0.296 & 0.037 \\
\hline$E 29$ & $\begin{array}{l}\text { Work } \\
\text { (PFD) }\end{array}$ & 0.401 & $0.044 * *$ \\
\hline$E 29$ & $\begin{array}{l}\text { Recreation } \\
\text { (PFD) }\end{array}$ & 0.382 & 0.006 \\
\hline
\end{tabular}

In total 19 correlations were found. The lowest was between $\mathrm{F} 3$ and sleeping $(r=0.290, p=0.048$ small $)$ and the highest

between $\mathrm{F} 16$ and headaches $(\mathrm{r}=.457, \mathrm{p}=0.001$ medium). VHI-9i subscales: $\mathrm{E}=$ emotional, $\mathrm{P}=$ physiological, $\mathrm{F}=$ functional.

NDI-G factors: $\mathrm{CF}=$ cognitive functioning, $\mathrm{PFD}=$ pain and functional disability. ${ }^{*}$ Correlation is significant at the 0.01 level.

* Correlation is significant at the 0.05 level.

\section{Gender}

There was a moderate statistically significant correlation between the overall VHI-9i and NDI-G for men $(\mathrm{r}=0.317, P=0.04)$, but not for women $(\mathrm{r}=0.192, P=0.14)$. In the male subgroup, there were six correlations higher than 0.300 , all representing a moderate relation. Three correlations were between the physiological item P4 of the VHI, one of a functional item (F16), and two of the emotional item (E29). The statistically strongest significant correlations were between "Reading" and P4 ( $\mathrm{r}=0.480, P=0.002)$, "Headaches" and P4 ( $\mathrm{r}=0.465, P=0.002)$, "Recreation" and P4 $(\mathrm{r}=0.479, P=0.002)$ and "Recreation" and E29 $(r=0.416, P=0.007)$. For a complete overview of all correlations of this subgroup, please refer to Table 4.

Table 4 Overview of All Correlations of the Male Subgroup

alt-text: Table 4 


\begin{tabular}{|l|l|l|l|}
\hline$P 4$ & Reading (PFD) & 0.480 & $.002^{* *}$ \\
\hline P4 & Headaches (CF) & 0.465 & $.002^{* *}$ \\
\hline F16 & Headaches (CF) & 0.316 & .044 \\
\hline P4 & Recreation (PFD) & 0.479 & $.002^{* *}$ \\
\hline$E 29$ & Work (PFD) & 0.330 & .035 \\
\hline$E 29$ & Recreation (PFD) & 0.416 & $.007^{* *}$ \\
\hline
\end{tabular}

Recreation (PFD)

0.416

Six correlations were found in total. The lowest between F16 and headaches $(\mathrm{r}=0.316, P=0.044)$ and the highest between $P$ and reading $(r=0.480, p=0.002)$. VHI-9i subscales: $E=$ emotional, $P=$ physiological, $F=$ functional. NDI-G factors:

$\mathrm{CF}=$ cognitive functioning, $\mathrm{PFD}=$ pain and functional disability. $* *$ Correlation is significant at the 0.01 level. * Correlation is significant at the 0.05 level.

Age

In patients below 60 years there was a weak and not significant correlation $(\mathrm{r}=0.149, P=0.22)$ between neck and voice-related subjective symptoms. A weak and not significant relation was also observed in older patients over 60 years $(\mathrm{r}=0.287, P=0.11)$

\section{Discussion}

In the present study of 50 patients with functional dysphonia and organic voice disorders each, there was a mild correlation between voice and neck-related subjective symptoms mainly in patients with organic voice disorders. This indicates an increased risk for a functional imbalance of the muscles surrounding the larynx in patients with structural pathology, which in extreme cases may hinder functional voice rehabilitation even after surgical treatment.

\section{Do women or men have more neck-related symptoms?}

Silverio and colleagues investigated in 30 vocally healthy women and 30 women with voice disorders in which body area they had additional pain, and how often they experienced it. ${ }^{13}$ The dysphonic women reported pain more often in the back of the neck than in any other body region. Furthermore, the pain intensity was higher. Based on this it was suggested that women with dysphonia have more changes in the cervical range of motion, pain while moving and palpation of the neck, and damaged cervical joint function. ${ }^{13}$

In the present study, we focused on subjective disability during daily life activities additionally to neck pain. $45.8 \%$ of women had moderate to severe neck disability, while in average the men did not present with such intense neck dysfunction. However, there was a statistically significant moderate correlation with subjective vocal handicap for male, but not for female patients. One explanation for this may be that men tend 
to seek medical help later as compared to women. ${ }^{34}$ Therefore, their individual voice problems might have already had a bigger impact at the first assessment. This might also explain why men presented with more structural lesions, which have been discussed to occur as a consequence of prolonged vocal misuse. ${ }^{35}$

\section{Neck disability in organic and functional voice disorders}

In our study, patients with organic voice disorders had a significant and weak (nearly moderate) correlation of voice and neck-related symptoms, which was stronger as compared to patients with functional voice disorders. In our sample, there were mainly patients with structural findings such as cysts, edema, or granulomas (47 out of 50, Table 1). Due to structural alterations changing the vibratory properties of the vocal folds, these patients may be forced to use a different voice technique in order to meet daily voice demands. This might result in an adaptation phenomenon with different recruitment patterns of the muscles moving and surrounding the larynx. ${ }^{35}$ A similar effect has been described in patients with shoulder disorders, who tend to alter scapula movement patterns to avoid pain. ${ }^{36}$ While this strategy may be useful for some time, it can lead to an overuse of specific muscles. In our sample the prevalence of neck pain was around $40 \%$, which is much higher than the reported values of $5 \%$ in the general population. ${ }^{37}$ Even though our methods using a single questionnaire might not be fully representative, this substantiates that a functional imbalance of the muscles surrounding the larynx may accompany organic and especially structural voice disorders. ${ }^{8}$

As discussed before, structural lesions may occur as a consequence of prolonged vocal misuse. ${ }^{8,35}$ Tomlinson and Archer (2015) argued that treatment approaches targeting the neck muscles may be efficient in treating specific voice disorder types. ${ }^{38}$ Also Mathieson proposed based on a literature review, that manual therapy may be an efficient primary treatment approach in patients with functional dysphonia. ${ }^{21}$ In our sample, the association with neck dysfunction was not significant for the functional voice patient subgroup, showing that neck dysfunction may not be a main contributing factor for all patients with functional voice disorders. In addition, the present study design does not allow conclusions, if neck dysfunction was the underlying cause for voice dysfunction in patients with organic findings. However, as discussed above, neck dysfunction may occur as an adaptation pattern to pathology and therefore hinder functional voice rehabilitation. Guidelines of the American Physical Therapy Association and a recent review, focusing on the efficacy of treatment, recommend physical therapy, specifically manual therapy in combination with exercise for patients with neck pain. ${ }^{39,40}$ Therefore, a comprehensive voice assessment should also include a standardized screening of neck symptoms. Patients with a suspected cervical dysfunction as a potential cause or consequence of a voice disorder should be referred to specialists for musculoskeletal disorders and may profit from neck disorder- 


\section{Age effects}

The human larynx undergoes morphological changes in the mucosa, muscles, and cartilages with age, leading to perceptual and instrumental acoustic changes in the voice and a reduced voice capacity. However, especially the onset and nature of changes to voice sound and capacity have been subject to debate. ${ }^{41-45}$ Further, age effects seem to be individual and dependent on voice training or use. ${ }^{46}$ Similarly, age, especially $>45$ years, is a known factor influencing the onset, course and recurrence of neck pain with highly individual patterns. ${ }^{47-49}$ Especially a reduced cervical range of motion might be explained by musculoskeletal, vestibular central nervous processing and sensorimotor changes as well as by cartilage degeneration. ${ }^{50}$ In the present sample, older patients did not show a stronger relation between voice and neck-related subjective symptoms. The main focus of the patients in the present study were voice-related complaints, therefore subjective neck dysfunction as indicated by NDI-G might not have been as relevant to them. Furthermore, due to the above discussed large natural differences between individuals, our sample might have been too small to fully characterize age effects on subjective voice and neck dysfunction.

\section{Critical discussion of self-assessment-based investigations}

Besides the methodological challenge of large natural differences between individuals regarding subjective voice and neck-related symptoms, the present results have to be discussed in the light of general issues in the application of self-assessment tools. Some patients might be more aware of their problems than others at the assessment point in time, due to factors such as personality, general education level, medical history, or professional circumstances. ${ }^{51-54}$ In addition, subjective and objective medical findings may not agree, for which reason the representativity of patient-based questionnaires has been questioned. Even though voice-related questionnaires have been shown to capture independent diagnostic information, they might not assess all relevant aspects, because the questions are given and do not allow any refinement to the patientsspecific symptom patterns. Further studies with different patient groups including an objective assessment of neck dysfunction are to characterize, how neck dysfunction influences voice function in order to determine efficient diagnosis and treatment approaches in voice patients with neck disability.

\section{Conclusion}

There was a significant weak correlation between voice and neck complaints in patients with organic voice disorders. Further, men had a moderate relation between neck and voice symptoms, and presented more 
often with benign structural pathologies as compared to women. This indicates an increased risk for a functional imbalance of the muscles surrounding the larynx and of the neck in patients with organic voice disorders, which in extreme cases may hinder functional voice rehabilitation. Therefore, neck dysfunction should be considered in voice diagnostics and, if appropriate, a standardized neck-specific assessment and treatment should be added to voice therapy.

\section{Appendix 1}

\section{Voice Handicap Index-9 international (VHI-9i)}

(9-item broad range subset)

Name:

These are statements that many people have used to describe their voices and the effects of their voices on their lives. Check the response that indicates how frequently you have the same experience.
$0=$ never
$1=$ almost
never
$2=$ sometimes
$3=$ almost
always $4=$ always

F1) My voice makes it difficult for people to hear me.

01234

F3) People have difficulty understanding me in a noisy room.

01234

P4) The sound of my voice varies throughout the day.

01234

F5) My family has difficulty hearing me, when I call them throughout the house.

01234

F16) My voice difficulties restrict my personal and social life.

01234

P17) The clarity of my voice is unpredictable.

01234 
P21) My voice is worse in the evening.

01234

E24) I am less outgoing because of my voice problem.

01234

E29) My voice makes me feel incompetent.

01234

\section{Appendix 2 \\ Neck Disability Index}

This questionnaire is designed to help us better understand how your neck pain affects your ability to manage everyday-life activities. Please mark in each section the one box that applies to you. Although you may consider that two of the statements in any one section relate to you, please mark the box that most closely describes your present-day situation.

\section{SECTION 1 - PAIN INTENSITY}

I have no pain at the moment.

The pain is very mild at the moment.

The pain is moderate at the moment.

The pain is fairly severe at the moment.

The pain is very severe at the moment.

The pain is the worst imaginable at the moment.

\section{SECTION 2 - PERSONAL CARE}

I can look after myself normally without causing extra pain.

I can look after myself normally, but it causes extra pain.

It is painful to look after myself, and I am slow and careful. 
I need help every day in most aspects of self-care.

I do not get dressed. I wash with difficulty and stay in bed.

\section{SECTION 3 - LIFTING}

I can lift heavy weights without causing extra pain.

I can lift heavy weights, but it gives me extra pain.

Pain prevents me from lifting heavy weights off the floor but I can manage if items are conveniently positioned, ie on a table.

Pain prevents me from lifting heavy weights, but I can manage light weights if they are conveniently positioned.

I can lift only very light weights.

\section{SECTION 4 - WORK}

I can do as much work as I want.

I can only do my usual work, but no more.

I can do most of my usual work, but no more.

I can't do my usual work.

I can hardly do any work at all.

I can't do any work at all.

\section{SECTION 5 - HEADACHES}

I have no headaches at all.

I have slight headaches that come infrequently.

I have moderate headaches that come infrequently.

I have moderate headaches that come frequently.

I have severe headaches that come frequently. 
I have headaches almost all the time.

\section{SECTION 6 - CONCENTRATION}

I can concentrate fully without difficulty.

I can concentrate fully with slight difficulty.

I have a fair degree of difficulty concentrating.

I have a lot of difficulty concentrating.

I have a great deal of difficulty concentrating.

I can't concentrate at all.

\section{SECTION 7 - SLEEPING}

I have no trouble sleeping.

My sleep is slightly disturbed for less than 1 hour.

My sleep is mildly disturbed for up to 1-2 hours.

My sleep is moderately disturbed for up to 2-3 hours.

My sleep is greatly disturbed for up to 3-5 hours.

My sleep is completely disturbed for up to 5-7 hours.

\section{SECTION 8 - DRIVING}

I can drive my car without neck pain.

I can drive as long as I want with slight neck pain.

I can drive as long as I want with moderate neck pain.

I can't drive as long as I want because of moderate neck pain.

I can hardly drive at all because of severe neck pain.

I can't drive my car at all because of neck pain.

I cannot lift or carry anything at all. 


\section{SECTION 9 - READING}

I can read as much as I want with no neck pain.

I can read as much as I want with slight neck pain.

I can read as much as I want with moderate neck pain.

I can't read as much as I want because of moderate neck pain.

I can't read as much as I want because of severe neck pain.

I can't read at all

\section{SECTION 10 - RECREATION}

I have no neck pain during all recreational activities.

I have some neck pain with all recreational activities.

I have some neck pain with a few recreational activities.

I have neck pain with most recreational activities.

I can hardly do recreational activities due to neck pain.

I can't do any recreational activities due to neck pain.

\section{PATIENT NAME_ DATE_}

\section{SCORE _ [50] BENCHMARK -5 =}

Copyright: Vernon H. and Hagino C., 1987. Vernon H, Mior S. The Neck Disability Index: A study of reliability and validity. Journal of Manipulative and Physiological Therapeutics 1991; 14:409-415. Copied with permission of the authors.

\section{References}

1. SR Schwartz, SM Cohen, SH Dailey, et al., Clinical practice guideline: hoarseness (dysphonia),

Otolaryngol - Head Neck Surg 141 (3 Suppl. 2), 2009, S1-S31,

https://doi.org/10.1016/j.otohns.2009.06.744.

2. KH Kim, RB Kim, DU Hwang, et al., Prevalence of and sociodemographic factors related to voice

disorders in South Korea, J Voice 30, 2016, https://doi.org/10.1016/j.jvoice.2015.04.010, 246e1-246e7. 
3. RHG Martins, HA do Amaral, ELM Tavares, et al., Voice disorders: etiology and diagnosis, J Voice 2015, https://doi.org/10.1016/j.jvoice.2015.09.017.

4. A Meulders and JWS Vlaeyen, Fear reduction in subacute whiplash-associated disorders: the royal road to recovery, Pain 154, 2013, 330-331, https://doi.org/10.1016/j.pain.2013.01.004.

5. EV Houtte, S Claeys, F Wuyts, et al., The impact of voice disorders among teachers : vocal complaints, treatment-seeking behavior, knowledge of vocal care, and voice-related absenteeism, J Voice 25, 2011, 570-575, https://doi.org/10.1016/j.jvoice.2010.04.008.

6. E Van Houtte, K Van Lierde, E D'Haeseleer, et al., The prevalence of laryngeal pathology in a treatmentseeking population with dysphonia, Laryngoscope 120, 2010, 306-312,

https://doi.org/10.1002/lary.20696.

7. CA Rosen and T Murry, Nomenclature of voice disorders and vocal pathology, Otolaryngol Clin North Am 33, 2000, 1035-1046, https://doi.org/10.1016/S0030-6665(05)70262-0.

8. E Van Houtte, K Van Lierde and S Claeys, Pathophysiology and treatment of muscle tension dysphonia: a review of the current knowledge, $J$ Voice 25, 2011, 202-207,

https://doi.org/10.1016/j.jvoice.2009.10.009.

9. T Nawka, IM Verdonck-De Leeuw, M De Bodt, et al., Item reduction of the voice handicap index based on the original version and on European translations, Folia Phoniatr Logop 61, 2009, 37-48, https://doi.org/10.1159/000200767.

10. AP Reimann, LTD Siqueira, AV Rondon, et al., Efeito imediato da terapia manual laríngea em indivíduos disfônicos, CoDAS 28, 2016, 59-65, https://doi.org/10.1590/2317-1782/20162015089.

11. L Mathieson, SP Hirani, R Epstein, et al., Laryngeal manual therapy: a preliminary study to examine its treatment effects in the management of muscle tension dysphonia, $J$ Voice 23, 2009, 353-366, https://doi.org/10.1016/j.jvoice.2007.10.002.

12. KC Alves Silverio, AG Brasolotto, L Thais Donalonso Siqueira, et al., Effect of application of transcutaneous electrical nerve stimulation and laryngeal manual therapy in dysphonic women: clinical trial, J Voice 29, 2015, 200-208, https://doi.org/10.1016/j.jvoice.2014.06.003.

13. KCA Silverio, LTD Siqueira, JRP Lauris, et al., Muscleskeletal pain in dysphonic women, CoDAS 26, 
14. SM. Cohen, Self-reported impact of dysphonia in a primary care population: an epidemiological study, Laryngoscope 120, 2010, 2022-2032, https://doi.org/10.1002/lary.21058.

15. T Vos, C Allen, M Arora, et al., Global, regional, and national incidence, prevalence, and years lived with disability for 310 diseases and injuries, 1990-2015: a systematic analysis for the Global Burden of Disease Study 2015, Lancet 388, 2016, 1545-1602, https://doi.org/10.1016/S0140-6736(16)31678-6.

16. DG Hoy, M Protani, R De, et al., The epidemiology of neck pain, Best Pract Res Clin Rheumatol 24, 2010, 783-792, https://doi.org/10.1016/j.berh.2011.01.019.

17. N Bogduk, The anatomy and pathophysiology of neck pain, Phys Med Rehabil Clin N Am 22, 2011, 367-382, https://doi.org/10.1016/j.pmr.2011.03.008.

18. R. Rao, Neck Pain, Cervical Radiculopathy, and cervical myelopathy: pathophysiology, natural history, and clinical evaluation, J Bone Joint Surg 84-A, 2002, 479-488 http://www.ncbi.nlm.nih.gov/pubmed/21137285.

19. R Ferrari and AS Russell, Neck pain, Best Pract Res Clin Rheumatol 17, 2003, 57-70, https://doi.org/10.1053/yberh.2003.269.

20. SL Smith and IR Titze, Characterization of flow-resistant tubes used for semi-occluded vocal tract voice training and therapy, J Voice 31, 2017, https://doi.org/10.1016/j.jvoice.2016.04.001, 113.e1-113.e8.

21. L Mathieson, The evidence for laryngeal manual therapies in the treatment of muscle tension dysphonia, Curr Opin Otolaryngol Head Neck Surg 19, 2011, 171-176.

22. PH Dejonckere, PJ Bradley, P Clemente, et al., A basic protocol for functional assessment of voice pathology, especially for investigating the efficacy of (phonosurgical) treatment and evaluating new assessment techniques, Eur Arch Otorhinolaryngol 258, 2001, 77-82, https://doi.org/10.1007/s004050000299.

23. T Nawka, U Wiesmann and U Gonnermann, Validation of the German version of the voice handicap index, HNO 51, 2003, 921-930, https://doi.org/10.1007/s00106-003-0909-8.

24. T Nawka, IM Verdonck-de Leeuw, M De Bodt, et al., Item reduction of the voice handicap index based on the original version and on European translations, Folia Phoniatr Logop 61, 2009, 37-48, 
25. CA Rosen, Lee AS, J Osborne, et al., Development and validation of the voice handicap index-10, Laryngoscope 114, 2004, 1549-1556, https://doi.org/10.1097/00005537-200409000-00009.

26. PN Carding, JA Wilson, K MacKenzie, et al., Measuring voice outcomes: state of the science review, $J$ Laryngol Otol 123, 2009, 823-829, https://doi.org/10.1017/S0022215109005398.

27. M Brockmann-Bauser and JE Bohlender, Forum Logopädie; Praktische Stimmdiagnostik; Theoretischer Und Praktischer Leitfaden, In: D Schrey-Dern and N Lauer, (Eds.), 2014, Georg Thieme Verlag; Stuttgart, doi:978-3-13-170921-9.

28. B Wiitavaara and M Heiden, Content and psychometric evaluations of questionnaires for assessing physical function in people with neck disorders: a systematic review of the literature, Disabil Rehabil $\mathbf{0}$, 2017, 1-9, https://doi.org/10.1080/09638288.2017.1334096.

29. H Vernon and S Mior, The neck disability index: a study of reliability and validity, J Manipulative Physiol Ther 14, 1991, 409-415.

30. J Swanenburg, K Humphreys, A Langenfeld, et al., Validity and reliability of a German version of the neck disability index (NDI-G), Man Ther 19, 2014, 52-58, https://doi.org/10.1016/j.math.2013.07.004.

31. H Vernon, The neck disability index: state-of-the-art, 1991-2008, J Manipulative Physiol Ther 31, 2008, 491-502, https://doi.org/10.1016/j.jmpt.2008.08.006.

32. GR Norman and DL Streiner, Biostatistics-The Bare Essentials, 3rd ed., 2008, People's Medical Publishing House; Shelton.

33. A. Field, Discovering Statistics Using IBM SPSS Statistics, In: M Carmicheal, (Ed), 4th ed., 2013, SAGE Publications Ltd.

34. RM Pinkhasov, J Wong, J Kashanian, et al., Are men shortchanged on health? Perspective on health care utilization and health risk behavior in men and women in the United States, Int J Clin Pract 64, 2010, 475-487, https://doi.org/10.1111/j.1742-1241.2009.02290.x.

35. J Bohlender, Diagnostic and therapeutic pitfalls in benign vocal fold diseases, GMS Curr Top Otorhinolaryngol Head Neck Surg 12, 2013, https://doi.org/10.3205/cto000093, Doc01.

36. JJ Lin, WP Hanten, SL Olson, et al., Functional activity characteristics of individuals with shoulder 
https://doi.org/10.1016/j.jelekin.2005.01.006.

37. D Hoy, L March, A Woolf, et al., The global burden of neck pain: estimates from the global burden of disease 2010 study, Ann Rheum Dis 73, 2014, 1309-1315, https://doi.org/10.1136/annrheumdis-2013204431.

38. CA Tomlinson and KR Archer, Manual therapy and exercise to improve outcomes in patients with muscle tension dysphonia: a case series, Phys Ther 95, 2015, 117-128, https://doi.org/10.2522/ptj.20130547.

39. B Hidalgo, T Hall, J Bossert, et al., The efficacy of manual therapy and exercise for treating non-specific neck pain : a systematic review, J Back Musculoskeletal Rehabil 30, 2017, 1149-1169, https://doi.org/10.3233/BMR-169615.

40. PR Blanpied, AR Gross, JM Elliott, et al., Neck pain : Revision 2017 clinical practice guidelines linked to the international classification of functioning, disability and health from the orthopaedic section of the American physical therapy association, J Orthop Sport Phys Ther 47, 2017, A1-A83, https://doi.org/10.2519/jospt.2017.0302.

41. RT Sataloff, DC Rosen, M Hawkshaw, et al., The aging adult voice, J Voice 11, 1997, 156-160, https://doi.org/10.1016/S0892-1997(97)80072-0.

42. RJ. Baken, The aged voice: a new hypothesis, J Voice 19, 2005, 317-325, https://doi.org/10.1016/j.jvoice.2004.07.005.

43. M Berg, M Fuchs, K Wirkner, et al., The speaking voice in the general population: normative data and associations to sociodemographic and lifestyle factors, $J$ Voice 31, 2018, https://doi.org/10.1016/j.jvoice.2016.06.001, 257.e13-257.e24.

44. RHG Martins, TM Gonçalvez, ABB Pessin, et al., Aging voice: presbyphonia, Aging Clin Exp Res 26 2014, 1-5, https://doi.org/10.1007/s40520-013-0143-5.

45. K Kendall, Presbyphonia: a review, Curr Opin Otolaryngol Head Neck Surg 15, 2007 https://journals.lww.com/co-otolaryngology/Fulltext/2007/06000/Presbyphonia_a_review.2.aspx.

46. S Reetz, JE Bohlender and M Brockmann-Bauser, Do standard instrumental acoustic, perceptual, and subjective voice outcomes indicate therapy success in patients with functional dysphonia, J Voice 2018, https://doi.org/10.1016/j.jvoice.2017.11.014. 
47. LJ Carroll, S Hogg-Johnson, GVD Velde, et al., Course and prognostic factors for neck pain in the general population neck pain and its associated disorders, Spine (Phila Pa 1976) 33, 2008, 75-82.

48. J Hill, M Lewis, AC Papageorgiou, et al., Predicting persistent neck pain: a 1-year follow-up of a population cohort, Spine (Phila Pa 1976) 29, 2004, 1648-1654

http://www.ncbi.nlm.nih.gov/pubmed/15284511.

49. A Langenfeld, BK Humphreys, J Swanenburg, et al., Prognostic factors for recurrences in neck pain patients up to 1 year after chiropractic care, J Manipulative Physiol Ther 2015, 1-7, https://doi.org/10.1016/j.jmpt.2015.06.014.

50. D Niederer, L Vogt, J Wilke, et al., Age-related cutoffs for cervical movement behaviour to distinguish chronic idiopathic neck pain patients from unimpaired subjects, Eur Spine J 24, 2015, 493-502, https://doi.org/10.1007/s00586-014-3715-y.

51. E Canals-Fortuny and J Vila-Rovira, Influence of self-generated anchors on the voice handicap index-10 (VHI-10), J Voice 31, 2018, https://doi.org/10.1016/j.jvoice.2016.06.011, 255.e9-255.e12.

52. AL Spina and AN Crespo, Assessment of grade of dysphonia and correlation with quality of life protocol, J Voice 31, 2018, https://doi.org/10.1016/j.jvoice.2016.04.005, 243.e21-243.e26.

53. M Behlau, F Zambon, F Moreti, et al., Voice self-assessment protocols: different trends among organic and behavioral dysphonias, J Voice 31, 2018, https://doi.org/10.1016/j.jvoice.2016.03.014, 112.e13-112.e27.

54. S Reetz, JE Bohlender and M Brockmann-Bauser, Do standard instrumental acoustic, perceptual, and subjective voice outcomes indicate therapy success in patients with functional dysphonia, $J$ Voice 2018, https://doi.org/10.1016/j.jvoice.2017.11.014. 